\title{
Noise measurement of a quantized charge pump
}

N. Maire, F. Hohls, B. Kaestner, K. Pierz, H. W. Schumacher, and R. J. Haug

Citation: Appl. Phys. Lett. 92, 082112 (2008); doi: 10.1063/1.2885076

View online: https://doi.org/10.1063/1.2885076

View Table of Contents: http://aip.scitation.org/toc/apl/92/8

Published by the American Institute of Physics

\section{Articles you may be interested in}

High-resolution error detection in the capture process of a single-electron pump

Applied Physics Letters 108, 023502 (2016); 10.1063/1.4939250

Gigahertz single-electron pumping in silicon with an accuracy better than 9.2 parts in $10^{7}$

Applied Physics Letters 109, 013101 (2016); 10.1063/1.4953872

Validation of a quantized-current source with $0.2 \mathrm{ppm}$ uncertainty Applied Physics Letters 107, 103501 (2015); 10.1063/1.4930142

Nanoampere charge pump by single-electron ratchet using silicon nanowire metal-oxide-semiconductor fieldeffect transistor

Applied Physics Letters 92, 042102 (2008); 10.1063/1.2837544

Robust single-parameter quantized charge pumping

Applied Physics Letters 92, 192106 (2008); 10.1063/1.2928231

Electron counting statistics and coherent states of electric current

Journal of Mathematical Physics 37, 4845 (1996); 10.1063/1.531672

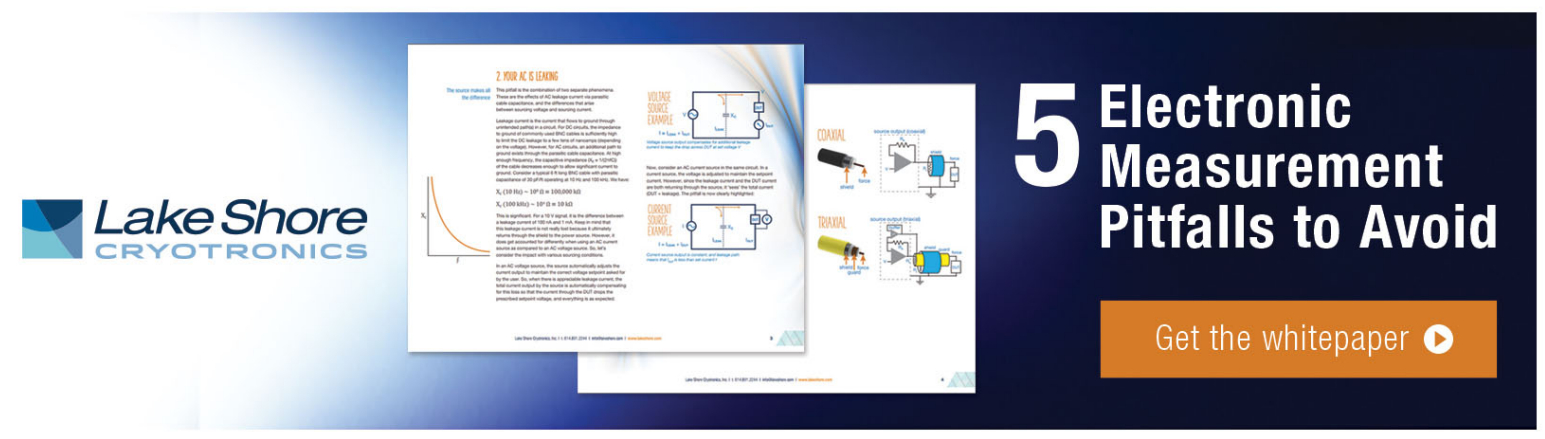




\title{
Noise measurement of a quantized charge pump
}

\author{
N. Maire, ${ }^{1, a)}$ F. Hohls, ${ }^{1}$ B. Kaestner, ${ }^{2}$ K. Pierz, ${ }^{2}$ H. W. Schumacher, ${ }^{2}$ and R. J. Haug ${ }^{1}$ \\ ${ }_{1}^{1}$ Institut für Festkörperphysik, Leibniz Universität Hannover, Appelstr. 2, 30167 Hannover, Germany \\ ${ }^{2}$ Physikalisch-Technische Bundesanstalt, Bundesallee 100, 38116 Braunschweig, Germany
}

(Received 10 December 2007; accepted 1 February 2008; published online 29 February 2008)

\begin{abstract}
We study the noise properties of a gate controlled single electron pump at a driving frequency $f_{p}$ $=400 \mathrm{MHz}$. We observe a significant reduction of the noise power on the current plateaus. This is a strong indication for true quantized charge pumping. We furthermore observe a small level of low frequency fluctuations which indicates a good frequency stability of the pump. (C) 2008 American Institute of Physics. [DOI: 10.1063/1.2885076]
\end{abstract}

Delivering a quantized number of electrons per cycle reliably and with a high repetition rate could allow to realize a quantum standard for the ampere. ${ }^{1-3}$ Several approaches to obtain such a quantized current pump have been tested. ${ }^{4-8}$ However, obtaining both a high current value and a high accuracy has proven to be difficult. Recently, Blumenthal et $a l$. have demonstrated a new promising way to deliver high currents. ${ }^{9}$ Their device consists of a semiconductor quantum wire crossed by three metal gates. For pumping, two of these gates are driven by phase locked high frequency signals. Such a pump allows gigahertz pumping comparable to surface-acoustic-wave pumps ${ }^{8}$ while promising higher accuracy.

Beside this application as a current standard, an electron pump could also be used as a fast on-demand electron source. Very recently, Feve et al. demonstrated the cyclic injection of coherent electrons using energy modulation of a quantum dot. ${ }^{10}$ Inherently, their device injects a quasihole between the injection of two electrons. A charge pump would however allow to inject a pure string of on-demand electrons.

In Ref. 9, the amplitude and phase relation of the two high frequency signals applied to the two gates have to be carefully controlled. Kaestner et al. have shown experimentally that it is sufficient to drive a single gate to achieve a quantized pumping current at very high frequency. ${ }^{11} \mathrm{~A}$ theoretical description of this nonadiabatic charge pumping leads to the conclusion that an optimized device could be suitable for metrological applications.

The standard criteria for quantized charge pumping used in all the aforementioned experiments is the appearance of current plateaus as a function of some control gate voltage with a quantized value $I=n e f_{p}$, where $f_{p}$ is the driving frequency, $e$ is the elementary charge, and $n$ is the integer number of electrons pumped in each cycle. However, for a quantized current pump used as a current standard, an independent proof of quantized charge pumping is necessary. Such independent proof can be delivered by a measurement of the current noise. The low frequency current noise $S_{I}(f)$ $=S_{I}^{0}$ for $f \ll f_{p}$ is expected to be frequency independent and becomes zero for an ideal quantized charge pump while a nonzero noise power $S_{I}^{0}$ reveals pumping errors. ${ }^{12-14}$

In this letter, we present noise measurements of a single gate quantized charge pump. We find that the current noise is

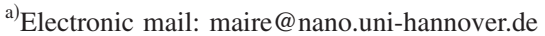

strongly suppressed at current plateaus with quantized values $I=n e f_{p}$ indicating quantized charge pumping. We also observe an enhanced current noise in the nonquantized current regions well described by a simple theoretical model. The level of $1 / f$ frequency fluctuations is low and restricted to $f \lesssim 1 \mathrm{kHz}$; for $f \geqq 2 \mathrm{kHz}$, the noise power is frequency independent as predicted.

The device used for our measurements is shown in Fig. 1(a). Three $100 \mathrm{~nm}$ wide metallic finger gates sit on top of a wire etched in a $n$-type AlGaAs heterostructure. The rightmost gate is grounded as it is not relevant for our measurements. A bound state in between the other two gates is formed by applying sufficiently large negative voltages $U 1$ and $U 2$ to both gates. An additional sinusoidal signal is then coupled to the left gate. If the oscillation amplitude is high enough, the bound state drops below the Fermi level during the first half-cycle of the periodic signal and can be loaded with electrons from the left reservoir. This is shown schematically by the solid curve in Fig. 1(b). During the second half-cycle the bound state is raised sufficiently fast to avoid backtunneling and electrons can be unloaded to the right (dashed curve) - a current is driven through the sample without an applied bias. The device acts as a quantized charge

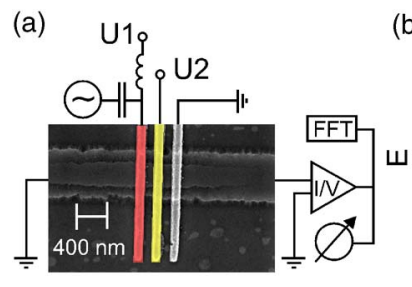

(b)

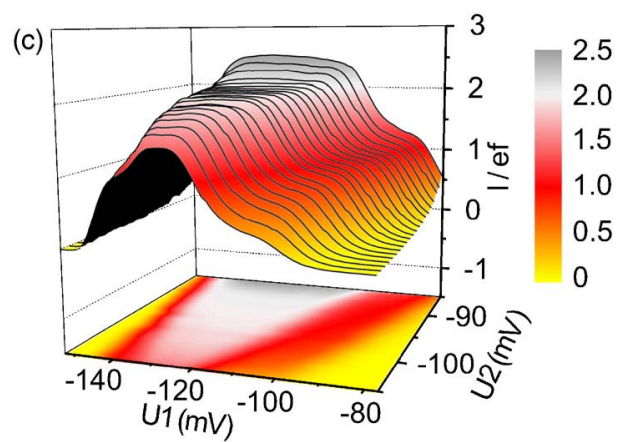

FIG. 1. (Color online) (a) SEM picture and measurement setup of the quantized charge pump. (b) Schematic of the pumping mechanism. (c) Detailed characteristic of the sample near the $2 e f$-plateau region. 

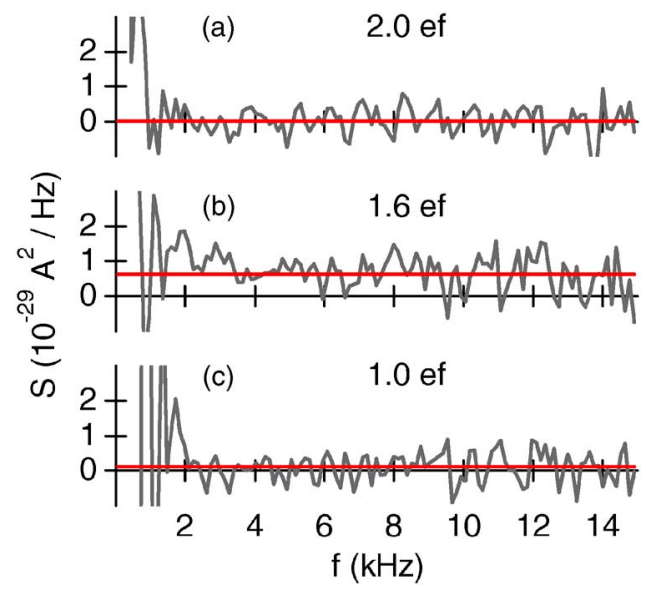

FIG. 2. (Color online) Three different noise spectra at voltages $U 1=$ $-125 \mathrm{mV}$ (a), $-103 \mathrm{mV}$ (b), and $-91 \mathrm{mV}$ (c). The horizontal lines correspond to the average noise power in the frequency range from 5 to $15 \mathrm{kHz}$ [(a) $0.16 \times 10^{-30} \mathrm{~A}^{2} / \mathrm{Hz}, \quad$ (b) $6.21 \times 10^{-30} \mathrm{~A}^{2} / \mathrm{Hz}, \quad$ and (c) 1.43 $\left.\times 10^{-30} \mathrm{~A}^{2} / \mathrm{Hz}\right]$.

pump. For a more detailed discussion of this pumping mechanism, we refer to Ref. 11.

The resulting current is converted into a voltage by a low noise current amplifier at room temperature with a bandwidth of $20 \mathrm{kHz}$ and measured via a dc voltmeter. The current noise is monitored by a fast-Fourier-transform analyzer. The measurements are carried out in a ${ }^{3} \mathrm{He}$ system at a temperature of $0.4 \mathrm{~K}$.

The corresponding pumped current of the sample driven at a frequency $f_{p}=400 \mathrm{MHz}$ with a power of $1 \mathrm{dBm}$ is shown as a function of $U 1$ and $U 2$ in Fig. 1(c). Pumping sets in for voltages $U 1 \gtrsim-150 \mathrm{mV}$ as the bound state is able to drop below the Fermi level during the first half of the periodic signal and an electron can be loaded onto the bound state. A current starts to rise and a small shoulder at $I \simeq e f_{p}$ appears as one electron is pumped per cycle. Further increase of $U 1$ leads to a broad plateau with well quantized current $I=2 e f_{p}$, where now two electrons are loaded and unloaded per cycle. Increasing the gate voltage even more imposes a limit on the maximum number of electrons that can be transferred-the bound state is not lifted high enough to account for both electrons to be unloaded to the right. Only the highest energetic state participates in the pumping process and the current drops to a narrow $I=e f_{p}$ plateau with just one electron per cycle. For even higher voltages $U 1$ the current again drops to zero as no electron can be unloaded to the right. Such current plateaus can be observed over a wide range of voltages $U 2$. For nonquantized current values in between the plateaus the number of electrons per pump cycle fluctuates.

In parallel to recording the current, we also measure the corresponding current noise at each voltage point. The resulting raw spectra contain also contributions of the amplifier noise. They are determined by applying a sufficiently negative pinch off value $U 1$ (which ensures $I=0$ ) and are consecutively subtracted from the raw spectra.

In Fig. 2, we show three current noise spectra for different voltages $U 1$ and an applied voltage $U 2=-86 \mathrm{mV}$. Note that these and the following data were obtained on the same device but after a different cool down cycle compared to Fig. 1(c) leading to a slight shift of the gate voltages at which the current plateaus occur. Part (a) corresponds to the $I=2 e f_{p}$

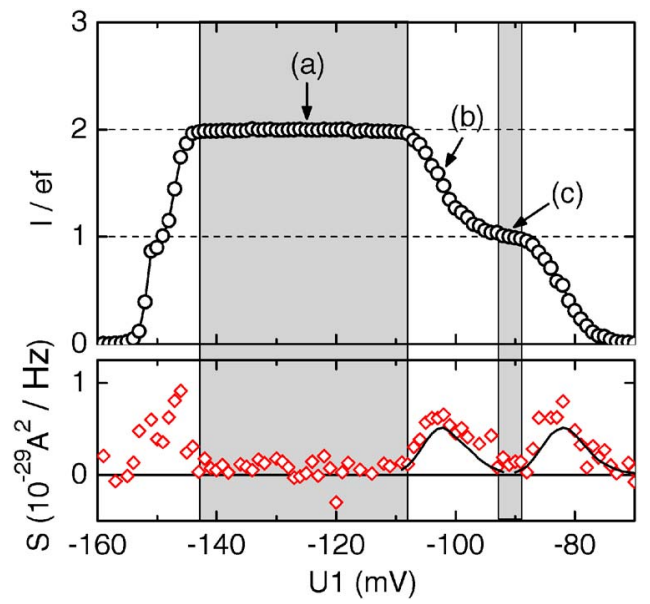

FIG. 3. (Color online) Top: Pumped current of the sample driven at $400 \mathrm{MHz}$ with a power of $1 \mathrm{dBm}$. Arrows (a), (b), and (c) indicate the voltage positions of the spectra shown in Fig. 2. Bottom: Corresponding noise power (symbols) averaged over the range of $5-15 \mathrm{kHz}(10-15 \mathrm{kHz}$ for $-152 \leqslant U 1 \leqslant-146$ ). The solid curves display theoretical estimates given by Eq. (3) (see text).

plateau at $U 1=-125 \mathrm{mV}$, (c) to the $I=1 e f_{p}$ plateau at $U 1=$ $-91 \mathrm{mV}$, and (b) shows the noise power in between at $U 1$ $=-103 \mathrm{mV}$ (see also Fig. 3). The horizontal lines equal noise powers averaged over the range of $5-15 \mathrm{kHz}$. The $1 / f$ low frequency noise drops at $1 \mathrm{kHz}$ already below $S$ $\sim 10^{-29} \mathrm{~A}^{2} / \mathrm{Hz}$; the current noise power $S$ is frequency independent for $f \gtrsim 2 \mathrm{kHz}$. This reveals a very good low frequency stability of the single gate quantized charge pump and confirms the prediction of white noise at frequencies $f$ $\ll f_{p}{ }^{12}$

Comparing the different spectra, we observe a very low noise level at the quantized current values - for spectra (a) and (c) the average noise power is $0.16 \times 10^{-30}$ and 1.4 $\times 10^{-30} \mathrm{~A}^{2} / \mathrm{Hz}$ - while the noise level in between these current plateaus at $U 1=-103 \mathrm{mV}$ [spectrum (b)] is considerably larger $\left(6.21 \times 10^{-30} \mathrm{~A}^{2} / \mathrm{Hz}\right)$. We will now analyze the level of white noise for the whole voltage range of $U 1$.

The average noise power as function of the gate voltage $U 1$ is displayed in the lower part of Fig. 3. The upper part shows the corresponding current. The slight $1 e f_{p}$ shoulder and the gray shaded quantized current plateau regions are clearly visible. In agreement with the spectra presented above, the noise power is strongly suppressed on these current plateaus compared to the noise level for nonquantized current values.

The current noise power of a quantized charge pump is directly linked to fluctuations in the number of electrons transported per pump cycle. If each cycle transports $n-1, n$ or $n+1$ electrons with probabilities $p_{n-1}, p_{n}$ and $p_{n+1}$ this leads to a noise power ${ }^{12-14}$

$$
S=2\left[p_{n-1}+p_{n+1}-\left(p_{n-1}-p_{n+1}\right)^{2}\right] e^{2} f_{p} .
$$

The pumped current is given by

$$
I=\left(n+p_{n+1}-p_{n-1}\right) e f_{p} .
$$

The combination of current and noise measurements then allows to fully characterize the probabilities $p_{n-1}, p_{n}$ and $p_{n+1}$. 
On the plateaus, the current is quantized, $I=n e f_{p}$. For an ideal quantized charge pump the probabilities to pump one less or one more electron $p_{n-1}$ and $p_{n+1}$ are both zero resulting in a noise power $S=0$ following Eq. (1).

The mean noise level, averaged over the measurements within the $I=2 e f_{p}$ plateau in Fig. 3 from $U 1=$ $-143 \mathrm{mV}$ to $U 1=-108 \mathrm{mV}$ is $\simeq 0.7 \times 10^{-30} \mathrm{~A}^{2} / \mathrm{Hz}$ which is on par with our present measurement uncertainty. From these measurements, we can thus estimate an upper limit of the total pumping error $\left(p_{n+1}+p_{n-1}\right)$ of about four percent.

We can additionally study the noise power in between the $I=2 e f_{p}$ and $I=1 e f_{p}$ current plateaus. It is reasonable to expect that the majority of pump cycles consists only of one or two electrons, $p_{1}+p_{2} \simeq 1$. The expected noise power is then given by

$$
S=2 p_{1}\left(1-p_{1}\right) e^{2} f_{p} .
$$

Using $I=\left(2-p_{1}\right) e f_{p}$, we can directly extract $p_{1}$ from the measured current and insert it into Eq. (3). The result is depicted by the solid curve in Fig. 3. We repeated this process for the voltage range in between zero and $I=1 e f_{p}$ current $\left(p_{0}+p_{1}\right.$ $\simeq 1, I=p_{1} e f_{p}$ ) and arrived at the same conclusion. The measured noise power fits well to the curve.

In conclusion, we have measured the current fluctuations of a single gate quantized charge pump. The pump shows a good low frequency stability and white noise power as predicted. We observe the expected quantized plateaus of the dc current. The noise measurements on these quantized current plateaus reveal a strong noise suppression thereby verifying quantized charge pumping. In addition, the measured noise level at nonquantized current values is in agreement with a simple theoretical model.
The authors would like to thank G. Hein, Th. Weimann, and H. Marx for support of clean room processing and sample growth. Funding was provided by the BMBF via project nanoQUIT.

${ }^{1}$ D. V. Averin and K. K. Lihkarev, J. Low Temp. Phys. 62, 345 (1986).

${ }^{2}$ I. M. Mills, P. J. Mohr, T. J. Quinn, B. N. Taylor, and E. R. Williams, Metrologia 43, 227 (2006).

${ }^{3}$ K. Flensberg, A. A. Odintsov, F. Liefrink, and P. Teunissen, Int. J. Mod. Phys. B 13, 2651 (1999).

${ }^{4}$ L. J. Geerligs, V. F. Anderegg, P. A. M. Holweg, J. E. Mooij, H. Pothier, D. Esteve, C. Urbina, and M. H. Devoret, Phys. Rev. Lett. 64, 2691 (1990).

${ }^{5}$ M. W. Keller, J. M. Martinis, N. M. Zimmerman, and A. H. Steinbach, Appl. Phys. Lett. 69, 1804 (1996).

${ }^{6}$ L. P. Kouwenhoven, A. T. Johnson, N. C. van der Vaart, C. J. P. M. Harmans, and C. T. Foxon, Phys. Rev. Lett. 67, 1626 (1991).

${ }^{7}$ H. Pothier, P. Lafarge, C. Urbina, D. Esteve, and M. H. Devoret, Europhys. Lett. 17, 249 (1992).

${ }^{8}$ J. M. Shilton, V. I. Talyanskii, M. Pepper, D. A. Ritchie, J. E. F. Frost, C. J. B. Ford, C. G. Smith, and G. A. C. Jones, J. Phys.: Condens. Matter 8, L531 (1996).

${ }^{9}$ M. D. Blumenthal, B. Kaestner, L. Li, S. Giblin, T. J. B. M. Janssen, M. Pepper, D. Anderson, G. Jones, and D. A. Ritchie, Nat. Phys. 3, 343 (2007).

${ }^{10}$ G. Feve, A. Mahe, J.-M. Berroir, T. Kontos, B. Placais, D. C. Glattli, A. Cavanna, B. Etienne, and Y. Jin, Science 316, 1169 (2007).

${ }^{11}$ B. Kaestner, V. Kashcheyevs, S. Amakawa, L. Li, M. D. Blumenthal, T. J. B. M. Janssen, G. Hein, K. Pierz, T. Weimann, U. Siegner, and H. W. Schumacher, e-print arXiv:cond-mat/07070993.

${ }^{12}$ Y. M. Galperin, O. Entin-Wohlman, and Y. Levinson, Phys. Rev. B 63, 153309 (2001).

${ }^{13}$ A. M. Robinson, V. I. Talyanskii, M. Pepper, J. E. Cunningham, E. H. Linfield, and D. A. Ritchie, Phys. Rev. B 65, 045313 (2002).

${ }^{14}$ A. M. Robinson and V. I. Talyanskii, Phys. Rev. Lett. 95, 247202 (2005). 